\title{
Quantum metric for null separated events and spacetime atoms
}

\author{
Alessandro Pesci \\ INFN Bologna, Via Irnerio 46, I-40126 Bologna, Italy
}

\begin{abstract}
Recently, a proposal has been made to figure out the expected discrete nature of spacetime at the smallest scales in terms of atoms of spacetime, capturing their effects through a scalar $\rho$, related to their density, function of the point $P$ and vector $v^{a}$ at $P$. This has been done in the Euclideanized space one obtains through analytic continuation from Lorentzian sector at $P$. $\rho$ has been defined in terms of a peculiar 'effective' metric $q_{a b}$, of quantum origin, introduced for spacelike/timelike separated events. This metric stems from requiring that $q_{a b}$ coincides with $g_{a b}$ at large (space/time) distances, but gives finite distance in the coincidence limit, and implements directly this way one single, very basic aspect associated to any quantum description of spacetime: length quantization. Since the latter appears a quite common feature in the available quantum descriptions of gravity, this quantum metric $q_{a b}$ can be suspected to have a rather general scope and to be re-derivable (and cross-checkable) in various specific quantum models of gravity, even markedly different one from the other.

This work reports on an attempt to introduce a definition of $\rho$ not through the Euclidean but directly in the Lorentz sector. This turns out to be not a so trivial task, essentially because of the null case, meaning when $v^{a}$ is null, as in this case it seems we lack even a concept of $q_{a b}$. A notion for the quantum metric $q_{a b}$ for null separated events is then proposed and an expression for it is derived. From it, a formula for $\rho$ is deduced, which turns out to coincide with what obtained through analytic continuation. This virtually completes the task of having quantum expressions of any kind of spacetime intervals, with, moreover, $\rho$ defined directly in terms of them (not in the Euclideanized space).
\end{abstract}

PACS numbers:

\section{STAYING IN THE LORENTZ SECTOR}

In the context of the attempts to provide a quantum theory of gravity or to describe spacetime quantum-

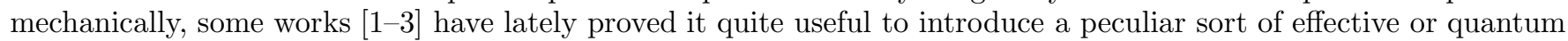
metric $q_{a b}$, also called qmetric, which acts to some extent as a metric at the same time allowing for the existence of a finite limiting distance $L$ between two events in their coincidence limit. It implements this way intrinsic discreteness of spacetime, still not abandoning the benefits, for calculus, associated to a continuous description of spacetime. One point of merit of this qmetric approach appears to be its genericity. Indeed, the quantum description it offers, does not come from a specific quantum theory of gravity but arises instead straight from simply requiring length quantization, a feature, this, one is likely to find in most specific models, and which has as such the status of quite a generic expectation when quantizing gravity. In Loop Quantum Gravity (LQG) [4 6 ] $]$ for example, quantization goes through the discretization of the classical theory (general relativity) and the introduction of a quantum theory associated to this discretization. We do get length quantization in it; this however not directly, but as a consequence of the general quantization procedure just mentioned. What we can say is that, concerning length quantization effects, it seems in principle we can compare what predicted by LQG and by the qmetric, with the predictions of the latter coming from length quantization without any specific theory associated with, and those of the former coming instead from the quantum framework provided by the specific theory. This means that the results one can obtain with the qmetric approach, could have wide range applicability within the various specific quantum gravity models, no matter how much they may differ one from the other in their starting assumptions and perspective (for example, whether the quantum theory of gravity has to come from the quantization of the classical theory of gravity or hinges instead on some, as yet unknown/untested, physics at Planck scale) and should be in principle recoverable in any one of them (and results in this sense have been reported in [7, 8] ).

One result one gets thanks to the quantum metric $q_{a b}$ is the possibility to provide a notion of degrees of freedom or of number of (quantum) states of spacetime at a point [9 12], fact which paves the way to a statistical description of field equations, and then to express the basic tenets of gravity using as proper language thermodynamics (as opposed

e-mail: pesci@bo.infn.it 
to geometry) [9]. This endows a previous statistical derivation of field equations [13, 14] and the notion of horizon microscopic degrees of freedom [15, 16] as well as recent results connecting so-called black hole chemistry [17, 18] with horizon degrees of freedom [19], all arising from macroscopic spacetime thermodynamics, with a microscopic mechanism seemingly able to directly justify these degrees of freedom. Key to the notion of degrees of freedom or of number of states of quantum spacetime is a quantity, denoted here $\rho$, defined in terms of $(D-1)$-dimensional areas (spacetime is assumed $D$-dimensional) of hypersurfaces formed by points at assigned distance from some point $P$ in the space coming from Euclideanisation of original spacetime around $P$. The basic feature about it is that, according to the effective metric, these $(D-1)$-areas remain finite in the coincidence limit of the hypersurfaces shrinking to $P$ [9] (and clearly, one would expect some analogous results do hold true in Lorentzian sector).

This of Euclideanisation might be a point of merit, providing insight perhaps into what the structure of the metric might be at the smallest scales. The procedure usually taken to go from a Lorentz signature to a Euclidean signature, the Wick rotation to imaginary time, even if well-established in flat spacetime, is however not free from ambiguities in general curved spacetime [20]. Based on a consistent prescription for this [20] (coming from reconsidering the Wick rotation as an analytic continuation of the metric), results concerning the curvature tensors, when migrating from Lorentzian to Euclidean signature spacetimes, have been presented 21], showing it is worth pursuing in this vein in a qmetric context.

On the other hand, it is not so clear which is the role of Euclidean manifolds at a fundamental level, meaning if at the smallest scale the reference manifold is really to be considered Euclidean instead of Lorentzian. There is, after all, no physical proof supporting a non-Lorentzian signature for spacetime. And the context in which a dynamical signature change is foreseen -from a fundamental Euclidean signature to the Lorentzian signature we see in the universe-, along the lines e.g. of [22, 23], has been pointed out to entail difficulties whose origin can be traced back to how, or how strongly, quantum field theory reacts to changes of signature in the underlying manifold (effects like production of infinite number of particles with infinite energy) [24]. Sound arguments have been also reported, based on the consideration of saddle-point approximation methods, showing that quantum gravity amplitudes should be defined first in terms of Lorentzian path integrals [25]. Likewise, from Wick rotation as analytic continuation of the metric, the suggestion has been given that the functional integral should be computed not over all Euclidean manifolds but only over those compatible with a Lorentz structure [20]. In causal dynamical triangulation, evidence has been reported indeed that one gains control on the functional integration if the sum is not taken over all Euclidean geometries, but is restricted instead only to those which are associated with Lorentzian causal geometries 26].

In view of all this, one would then know if the qmetric approach could allow to pick up quantum degrees of freedom even never abandoning the Lorentz sector. The aim of present study is precisely to develop a concept of $\rho$ in the Lorentz sector directly, i.e. with no reliance on Euclideanised space. A partial result in this direction has been already presented in 27]. There, a notion of $\rho$ for timelike geodesics has been introduced and its expression has been derived (and the case of spacelike geodesics goes along similar lines). What is left is the consideration of null geodesics and this is the case we try to study here. As we will see, this involves the introduction of a notion of quantum metric $q_{a b}$ for null separated events, this way completing a quantum formulation of spacetime intervals.

\section{II. $\rho$ FOR TIMELIKE/SPACELIKE GEODESICS}

Let us start by recalling what we can do with timelike/spacelike geodesics. We briefly rephrase what is reported in 27] for timelike case, using here a notation which encompasses both the timelike and the spacelike case at one stroke. We consider timelike/spacelike geodesics through a generic point $P$ in spacetime, and introduce the two hypersurfaces $\Sigma_{\epsilon}(P, l), \epsilon=+1$ for spacelike geodesics and $\epsilon=-1$ for timelike ones, of all points $p$ at assigned squared distance from $P$ :

$$
\Sigma_{\epsilon}(P, l)=\left\{p: \epsilon \sigma^{2}(p, P)=l^{2}\right\},
$$

where $\sigma^{2}(p, P)$ is the squared geodesic distance between $P$ and $p\left(\sigma^{2}(p, P)=2 \Omega(p, P)\right.$, with $\Omega(p, P)$ the Synge world function [28]), and $l=\sqrt{l^{2}}$ non-negative.

Proceeding analogously to the Euclidean definition, $\rho$ is given in terms of generic/flat ratio of element of areas on $\Sigma_{\epsilon}(P, l)$, as measured according to the effective metric, in the limit $l \rightarrow 0$. For each assigned normalised vector $n^{a}$ at $P\left(n^{a} n_{a}=\epsilon\right)$, we consider the intersection point $p$ between the geodesic $\mu\left(n^{a}\right)$ with tangent at $P t^{a}(P)=n^{a}$ and the hypersurface $\Sigma_{\epsilon}(P, l)$. Calling $y^{i}, i=1, \ldots, D-1$ coordinates on $\Sigma_{\epsilon}(P, l)$ such that $y^{i}(p)=0$, we consider a segment $I$ of hypersurface $\Sigma_{\epsilon}(P, l)$ around $p$, defined as $I=\left\{d y^{i}\right\}$, where $d y^{i}$ are thought as fixed when $l$ is varied. The $(D-1)$-dimensional area of $I$ is 


$$
d^{D-1} V(p)=\sqrt{-\epsilon h(p)} d^{D-1} y
$$

where $h_{i j}$ are the components of the metric on $\Sigma_{\epsilon}(P, l)$ in the coordinates $y^{i}$, metric which coincides with that induced by spacetime metric $g_{a b}$. What we have to consider is the area $\left[d^{D-1} V\right]_{q}$ of $I$ as measured through the effective metric $q_{a b}$.

The effective metric is described [1, 3] in terms of the bitensor $q_{a b}(p, P)$ which stems from requiring the squared geodesic distance $\sigma^{2}$ gets modified into $\sigma^{2} \rightarrow\left[\sigma^{2}\right]_{q}=S_{L}\left(\sigma^{2}\right)$ with $(\mathbf{R} 1) S_{0}=\sigma^{2},(\mathbf{R 2}) S_{L}\left(0^{ \pm}\right)= \pm L^{2}$, and $(\mathbf{R 3})$ the kernel $G\left(\sigma^{2}\right)$ of the d'Alembertian gets modified into $G\left(\sigma^{2}\right) \rightarrow[G]_{q}\left(\sigma^{2}\right)=G\left(S_{L}\right)$ in all maximally symmetric spacetimes. These requirements give, for spacelike or timelike geodesics, the expression

$$
q_{a b}(p, P)=A\left(\sigma^{2}\right) g_{a b}(p)+\epsilon\left(\frac{1}{\alpha\left(\sigma^{2}\right)}-A\left(\sigma^{2}\right)\right) t_{a}(p) t_{b}(p)
$$

where $t^{a}$ is the normalized tangent vector $\left(g_{a b} t^{a} t^{b}=\epsilon\right)$, not going to change its timelike or spacelike character when in the qmetric,

$$
\begin{gathered}
A=\frac{S_{L}}{\sigma^{2}}\left(\frac{\Delta}{\Delta_{S}}\right)^{\frac{2}{D-1}}, \\
\alpha=\frac{S_{L}}{\sigma^{2}\left(S_{L}^{\prime}\right)^{2}}
\end{gathered}
$$

(' indicates differentiation with respect to the argument $\sigma^{2}$ ), where

$$
\Delta(p, P)=-\frac{1}{\sqrt{g(p) g(P)}} \operatorname{det}\left[-\nabla_{a}^{(p)} \nabla_{b}^{(P)} \frac{1}{2} \sigma^{2}(p, P)\right]
$$

is the van Vleck determinant $(29-32]$; see $[33-35])$ which is a biscalar, and the biscalar $\Delta_{S}(p, P)$ is $\Delta_{S}(p, P)=$ $\Delta(\tilde{p}, P)$, where $\tilde{p}$ is that point on the geodesic through $P$ and $p$ (on the same side of $p$ with respect to $P$ ) which has $\sigma^{2}(\tilde{p}, P)=S_{L}(p, P)$. $\alpha$ is determined by the request that the formula for squared geodesic distance

$$
g^{a b} \partial_{a} \sigma^{2} \partial_{b} \sigma^{2}=4 \sigma^{2}
$$

(Hamilton-Jacobi equation) gets transformed into $q^{a b} \partial_{a} S_{L} \partial_{b} S_{L}=4 S_{L} ; A$ by the request R3.

From the effective metric $\left[h_{a b}\right]_{q}(p, P)$ induced by $q_{a b}(p, P)$ at $p$ on $\Sigma_{\epsilon}(P, l)$, we get the effective-metric $(D-1)$ dimensional area of $I$ as

$$
\left[d^{D-1} V\right]_{q}(p, P)=[\sqrt{-\epsilon h}]_{q}(p, P) d^{D-1} y
$$

As in the Euclidean approach, $\rho$ can then be defined as the ratio of effective-metric $(D-1)$-dimensional area of $I$ for the actual metric configuration, $\left[d^{D-1} V\right]_{q(g)}(p, P)$, to what we would have were spacetime flat, $\left[d^{D-1} V\right]_{q(\eta)}(p, P)\left(\eta_{a b}\right.$ is Minkowski metric), in the limit $p \rightarrow P$ along $\mu\left(n^{a}\right)$, i.e.

$$
\rho\left(P, n^{a}\right)=\left(\lim _{p \rightarrow P} \frac{\left[d^{D-1} V\right]_{q(g)}(p, P)}{\left[d^{D-1} V\right]_{q(\eta)}(p, P)}\right)_{\mu\left(n^{a}\right)} .
$$

$\rho$ is then derived in terms of the quantities $A$ and $\alpha$ defining the effective metric. The effective metric $\left[h_{a b}\right]_{q}$ induced by $q_{a b}$ turns out to be

$$
\left[h_{a b}\right]_{q}(p, P)=A\left(\sigma^{2}\right) h_{a b}(p)
$$

[36], which implies 


$$
[\sqrt{-\epsilon h}]_{q}(p, P)=A\left(\sigma^{2}\right)^{\frac{D-1}{2}} \sqrt{-\epsilon h(p)}
$$

and then

$$
\left[d^{D-1} V\right]_{q}(p, P)=A\left(\sigma^{2}\right)^{\frac{D-1}{2}} d^{D-1} V(p),
$$

where $d^{D-1} V(p)$ indicates the proper area of $I$ according to the ordinary metric. Here we see that only $A$, and not $\alpha$, is actually involved in the determination of $\rho$.

Introducing on $\Sigma_{\epsilon}$, in a neighbourhood of $p$, mutually orthogonal coordinates $z^{i}$ such that, chosen any one of them, $z^{\bar{i}}$, it can be written in the form $z^{\bar{i}}=l \eta$ with the parameter $\eta$ such that $l d \eta$ is proper distance or proper-time difference, and chosing as $I$ the (hyper)cube $d z^{i}$ defined by $d z^{i}=l d \eta, \forall i$, we obtain

$$
\left[d^{D-1} V\right]_{q}(p, P)=A\left(\sigma^{2}\right)^{\frac{D-1}{2}} l^{D-1}\left(1+\mathcal{O}\left(l^{2}\right)\right)(d \eta)^{D-1}
$$

where the $\mathcal{O}\left(l^{2}\right)$ term represents the effects of curvature (and is thus of course absent in flat case), and clearly $l=\sqrt{\epsilon \sigma^{2}}$. Using the expression (2) for $A$, we get

$$
\left[d^{D-1} V\right]_{q}(p, P)=\left[\epsilon S_{L}\right]^{\frac{D-1}{2}} \frac{\Delta(p, P)}{\Delta_{S}(p, P)}\left(1+\mathcal{O}\left(l^{2}\right)\right)(d \eta)^{D-1}
$$

and, in the limit $p \rightarrow P$ along $\mu\left(n^{a}\right)$,

$$
\lim _{l \rightarrow 0}\left[d^{D-1} V\right]_{q}(p, P)=L^{D-1} \frac{1}{\Delta_{L}\left(P, n^{a}\right)}(d \eta)^{D-1},
$$

with $\Delta_{L}\left(P, n^{a}\right)=\Delta(\bar{p}, P)$, where $\bar{p}$ is that point on geodesic $\mu\left(n^{a}\right)$ (on the side in the direction $n^{a}$ ) which has $l=L$.

This shows that both the numerator and the denominator in expression (6) remain non vanishing in the coincidence limit $p \rightarrow P$, exactly as it happens in Euclidean case. Since for flat spacetime $\Delta=1$ identically and then also $\Delta_{L}=1$, we have finally

$$
\rho\left(P, n^{a}\right)=\frac{1}{\Delta_{L}\left(P, n^{a}\right)},
$$

where the $\Delta_{L}$ is that of generic metric $g_{a b}$. The scope of this exact expression for $\rho$ clearly includes strictly Riemannian manifolds (as that from Euclideanisation).

Expanding $\Delta(p, P)$ in powers of $l([31] ;[33[35])$,

$$
\Delta(p, P)=1+\frac{1}{6} l^{2} R_{a b} t^{a} t^{b}+o\left(l^{2} R_{a b} t^{a} t^{b}\right),
$$

$\left(t^{a} t_{a}=\epsilon\right)$ gives

$$
\Delta_{L}\left(P, n^{a}\right)=1+\frac{1}{6} L^{2} R_{a b}(P) n^{a} n^{b}+o\left(L^{2} R_{a b}(P) n^{a} n^{b}\right)
$$

and

$$
\rho\left(P, n^{a}\right)=1-\frac{1}{6} L^{2} R_{a b}(P) n^{a} n^{b}+o\left(L^{2} R_{a b}(P) n^{a} n^{b}\right) .
$$

Again, this identically applies also to Riemannian manifolds (as that from Euclideanisation), and its form coincides with the expansion obtained [9-12] defining $\rho$ in the Euclideanised space. 


\section{QMETRIC AND NULL GEODESICS}

If we try to extend the scope of effective metric approach to include null geodesics, we have that expression (1) becomes ill defined in this case since $\sigma^{2}=0$ all along any null geodesic, and in principle we are then in trouble. We notice however the following. Any affine parametrization $\lambda$ of a null geodesic can be thought of as a measure of distance along the geodesic performed by a canonical observer picked up at a certain point $x$ of the geodesic and parallel transported along the geodesic. Since, when going to the effective metric $q_{a b}$, the squared distance in the coincidence limit is the finite value $\epsilon L^{2}$ (request $\mathbf{R} 2$ above), we could expect the effect of the qmetric in the null case is to induce a mapping of the parametrization $\lambda$ to a new parametrization $\tilde{\lambda}=\tilde{\lambda}(\lambda)$, with $\tilde{\lambda} \rightarrow L$ when $\lambda(p, P) \rightarrow 0$, i.e. when $p \rightarrow P$. In analogy with the spacelike/timelike case, we can then think to give an expression for $q_{a b}(p, P)$ when $p$ is on a null geodesic from $P$ in terms of two functions $\alpha_{\gamma}=\alpha_{\gamma}(\lambda)$ and $A_{\gamma}=A_{\gamma}(\lambda)$ defined on the geodesic, and determined by a condition on the squared geodetic distance and on the d'Alembertian. In other words, this suggests we assume that the effects of the existence of a limiting length are captured by an effective metric bitensor $q_{a b}$ as above, with its expression on a null geodesic stemming from requiring the affine parametrization $\lambda$ gets modified into $\lambda \rightarrow[\lambda]_{q}=\tilde{\lambda}(\lambda)$ with (G1) $\tilde{\lambda}=\lambda$ if $L=0$ (or $\tilde{\lambda} \simeq \lambda$ when $\left.\lambda \rightarrow \infty\right)$, (G2) $\tilde{\lambda}\left(0^{+}\right)=L$, and (G3) the kernel $G\left(\sigma^{2}\right)$ gets modified into $[G]_{q}\left(\sigma^{2}\right)=G\left(S_{L}\right)$ in all maximally symmetric spacetimes, i.e (G3) coincides with (R3) above on null geodesics.

We see that dealing with the null case appears quite not so obvious, in that we are forced to rewrite for this case from scratch the rules to go to the qmetric given a metric, in terms of an affine parameter $\lambda$ defined on null geodesics only, i.e. $q_{a b}$ is defined strictly on null geodesics and knows nothing outside them. And this, morover, leads to the tricky circumstances that the operators we look at when constraining the expression for $q_{a b}$ (e.g. the d'Alembertian) should be considered in a form which does not hinge on any knowledge, regarding the elements which enter the definition of the operator itself (directional derivatives, vectors), of what happens outside the $(D-1)$-dimensional submanifold swept by all the null geodesics emanating from a point.

Let $\gamma$ be a null geodesic through $P$, with affine parameter $\lambda=\lambda(p, P)$ with $\lambda(P, P)=0$, and null tangent vector $l^{a}=\frac{d x^{a}}{d \lambda}$, i.e. $\nabla_{a}\left(\sigma^{2}\right)=2 \lambda l_{a}$ (see e.g. [35]). We introduce a canonical observer at $P$, with velocity $V^{a}$, such that $l_{a} V^{a}=-1$. By parallel transport of the observer along $\gamma$, this relation extends all along $\gamma$, with $\lambda$ having the meaning of a distance as measured by this observer. We affinely parametrize any other null geodesic $\hat{\gamma}$ which goes through $P$, and require $\hat{l}_{a} V^{a}=-1$. What we obtain this way, is a $(D-1)$-dimensional congruence $\Gamma$ of null geodesics emanating from $P$ which is affinely parametrized and has deviation vectors orthogonal to the geodesics. We introduce a second null vector $m^{a}$ at $P$, defined by $m^{a} \equiv 2 V^{a}-l^{a}$, parallelly transported along the geodesic. This gives $m_{a} V^{a}=-1$ and $m_{a} l^{a}=-2$ all along $\gamma$. The vector $m^{a}$ does depend on the observer we have chosen.

Let $q_{a b}(p, P), p$ on $\gamma$, be of the form

$$
q_{a b}=A_{\gamma} g_{a b}-\frac{1}{2}\left(\frac{1}{\alpha_{\gamma}}-A_{\gamma}\right)\left(l_{a} m_{b}+m_{a} l_{b}\right)
$$

From $q^{a b} q_{b c}=\delta_{c}^{a}$, we get

$$
q^{a b}=\frac{1}{A_{\gamma}} g^{a b}+\frac{1}{2}\left(\frac{1}{A_{\gamma}}-\alpha_{\gamma}\right)\left(l^{a} m^{b}+m^{a} l^{b}\right),
$$

where $l^{a}=g^{a b} l_{b}, m^{a}=g^{a b} m_{b}$. Notice that $q^{a b} l_{a} l_{b}=0$, and the geodesic is null also according to the qmetric.

Our first task is to determine the form of $\alpha_{\gamma}$. To this aim, we use of the request that $\left[l^{a}\right]_{q}=d x^{a} / d \tilde{\lambda}$ be parallelly transported according to the qmetric. We need this, if $\tilde{\lambda}$ has to be interpreted as a (quantum) arc-length according to a canonical observer. We have

$$
\left[l^{b}\right]_{q}\left[\nabla_{b}\right]_{q}\left[l_{c}\right]_{q}=\frac{d \lambda}{d \tilde{\lambda}} l^{b}\left(\partial_{b}\left(\frac{d \lambda}{d \tilde{\lambda}} \frac{1}{\alpha_{\gamma}} l_{c}\right)-\left[\Gamma_{b c}^{a}\right]_{q} \frac{d \lambda}{d \tilde{\lambda}} \frac{1}{\alpha_{\gamma}} l_{a}\right)
$$

where $\left[l_{c}\right]_{q}=q_{a c}\left[l^{a}\right]_{q}=\frac{d \lambda}{d \tilde{\lambda}} \frac{1}{\alpha_{\gamma}} l_{c}$. Here, from $\Gamma_{b c}^{a}=\frac{1}{2} g^{a d}\left(-\partial_{d} g_{b c}+\partial_{c} g_{b d}+\partial_{b} g_{d c}\right)$, we have

$$
\begin{aligned}
{\left[\Gamma_{b c}^{a}\right]_{q} } & =\frac{1}{2} q^{a d}\left(-\partial_{d} q_{b c}+\partial_{c} q_{b d}+\partial_{b} q_{d c}\right) \\
& =\frac{1}{2} q^{a d}\left(-\nabla_{d} q_{b c}+2 \nabla_{(b} q_{c) d}\right)+\Gamma_{b c}^{a}
\end{aligned}
$$


(cf. [36]). Using of this, we get

$$
\begin{aligned}
{\left[l^{b}\right]_{q}\left[\nabla_{b}\right]_{q}\left[l_{c}\right]_{q} } & =\frac{d \lambda}{d \tilde{\lambda}} l_{c} \frac{d}{d \lambda}\left(\frac{d \lambda}{d \tilde{\lambda}} \frac{1}{\alpha_{\gamma}}\right)-\frac{1}{2}\left(\frac{d \lambda}{d \tilde{\lambda}}\right)^{2} l^{d} l^{b}\left(-\nabla_{d} q_{b c}+2 \nabla_{(b} q_{c) d}\right) \\
& =\frac{d \lambda}{d \tilde{\lambda}} l_{c} \frac{d}{d \lambda}\left(\frac{d \lambda}{d \tilde{\lambda}} \frac{1}{\alpha_{\gamma}}\right)-\left(\frac{d \lambda}{d \tilde{\lambda}}\right)^{2}\left(\frac{1}{\alpha_{\gamma}}-A_{\gamma}\right) l^{b} \nabla_{c} l_{b}
\end{aligned}
$$

where in the 1st equality we used of $l^{b} \nabla_{b} l_{c}=0$ and of $q^{a d} l_{a}=\alpha_{\gamma} l^{d}$, and, in the 2nd, of $l^{d} l^{b} \nabla_{c} q_{b d}=2\left(\frac{1}{\alpha_{\gamma}}-A_{\gamma}\right) l^{b} \nabla_{c} l_{b}$. Here, $\nabla_{c} l_{b}$ brings to consider variations of $l_{b}$ outside $\Gamma$. However, in whichever way might $l^{b}$ null be thougth to be extended outside $\Gamma$, always it will hold true that $\nabla_{c}\left(l^{b} l_{b}\right)=2 l^{b} \nabla_{c} l_{b}=0$. We have then

$$
\left[l^{b}\right]_{q}\left[\nabla_{b}\right]_{q}\left[l_{c}\right]_{q}=\frac{d \lambda}{d \tilde{\lambda}} l_{c} \frac{d}{d \lambda}\left(\frac{d \lambda}{d \tilde{\lambda}} \frac{1}{\alpha_{\gamma}}\right) .
$$

$\left[l^{b}\right]_{q}\left[\nabla_{b}\right]_{q}\left[l_{c}\right]_{q}=0$ requires $\alpha_{\gamma}=K \frac{d \lambda}{d \lambda}$, with $K$ a constant. To determine $K$ we use the following. When $\lambda \rightarrow \infty$, $d \lambda / d \tilde{\lambda} \rightarrow 1$ and we must have also $q_{a b} \rightarrow g_{a b}$. This implies both $\lim _{\lambda \rightarrow \infty} A_{\gamma}=1$ and $K=1$. What we get is thus

$$
\alpha_{\gamma}=\frac{1}{d \tilde{\lambda} / d \lambda}
$$

As for the determination of $A_{\gamma}$, we have to refer to $\mathbf{G 3}$, i.e we consider the d'Alembertian in maximally symmetric spaces at points on null geodesics. What we try first, is to find out some convenient expression for the d'Alembertian. Due to maximal symmetry, we can think in terms of $f=f\left(\sigma^{2}\right)$ and write

$$
\begin{aligned}
\square f & =\nabla_{a} \nabla^{a} f \\
& =\nabla_{a}\left(\partial^{a} \sigma^{2} \frac{d f}{d \sigma^{2}}\right) \\
& =\left(\nabla_{a} \partial^{a} \sigma^{2}\right) \frac{d f}{d \sigma^{2}}+\left(\partial^{a} \sigma^{2}\right) \partial_{a} \frac{d f}{d \sigma^{2}} \\
& =\left(\nabla_{a} \partial^{a} \sigma^{2}\right) \frac{d f}{d \sigma^{2}}+\left(\partial^{a} \sigma^{2}\right)\left(\partial_{a} \sigma^{2}\right) \frac{d^{2} f}{d\left(\sigma^{2}\right)^{2}} .
\end{aligned}
$$

When going to null geodesic $\gamma,\left(\partial^{a} \sigma^{2}\right)\left(\partial_{a} \sigma^{2}\right) \rightarrow\left(2 \lambda l^{a}\right)\left(2 \lambda l_{a}\right)=0$ and we get

$$
\square f=\left(\nabla_{a} \partial^{a} \sigma^{2}\right) \frac{d f}{d \sigma^{2}} .
$$

At a point $p^{\prime}$ close to $\Gamma$ but, possibly, not exactly on it, we can write (cf. [34])

$$
\partial^{a} \sigma_{\mid p^{\prime}}^{2}=2 \lambda l_{\mid p^{\prime}}^{a}+2 \nu m_{\mid p^{\prime}}^{a}
$$

where $\lambda$ and $\nu$ are curvilinear null coordinates of $p^{\prime}$ (there is a unique point $p$ on $\Gamma$ from which $p^{\prime}$ is reachable through a null geodesic $\beta$ with tangent $m^{a}$ at $p ; \nu$ is the affine parameter of $p^{\prime}$ along $\beta$, with $\left.\nu(p)=0\right), l_{\mid p^{\prime}}^{a}$ and $m_{\mid p^{\prime}}^{a}$ are $l^{a}$ and $m^{a}$ parallel transported along $\beta$ from $p$ to $p^{\prime}$. This gives, on $\gamma$,

$$
\begin{aligned}
\nabla_{a} \partial^{a} \sigma^{2} & =2\left(\lambda \nabla_{a} l^{a}+l^{a} \partial_{a} \lambda+m^{a} \partial_{a} \nu\right) \\
& =2\left(\lambda \nabla_{a} l^{a}+2\right),
\end{aligned}
$$

and then

$$
\square f=\left(4+2 \lambda \nabla_{a} l^{a}\right) \frac{d f}{d \sigma^{2}}=\left(4+2 \lambda \nabla_{i} l^{i}\right) \frac{d f}{d \sigma^{2}},
$$


$i=1, \ldots, D-1$ indices of components on $\Gamma$. Here, we emphasized the fact that, since the covariant derivative of $l^{a}$ along $\beta$ is $0, \nabla_{a} l^{a}$ is completely defined within $\Gamma$ and coincides with the expansion of $\Gamma, \nabla_{a} l^{a}=\nabla_{i} l^{i}$.

Going to the qmetric, the geodesic $\gamma$ remains null, and we have

$$
\begin{aligned}
{[\square f]_{q} } & =\left(4+2\left[\lambda \nabla_{a} l^{a}\right]_{q}\right)\left[\frac{d f}{d \sigma^{2}}\right]_{q} \\
& =\left(4+2[\lambda]_{q}\left[\nabla_{a} l^{a}\right]_{q}\right) \frac{d[f]_{q}}{d S_{L}} \\
& =\left(4+2 \tilde{\lambda}\left[\nabla_{a} l^{a}\right]_{q}\right)\left(\frac{d f}{d \sigma^{2}}\right)_{\mid \sigma^{2}=S_{L}} .
\end{aligned}
$$

Here $\left[l^{a}\right]_{q}=d x^{a} / d \tilde{\lambda}=(d \lambda / d \tilde{\lambda}) l^{a}$, and $f: \sigma^{2} \mapsto f\left(\sigma^{2}\right)$ gets mapped by the qmetric into $[f]_{q}: \sigma^{2} \mapsto S_{L} \mapsto f\left(S_{L}\right)=$ $[f]_{q}\left(\sigma^{2}\right)$ which has $\frac{d[f]_{q}}{d S_{L}}=\left(\frac{d f}{d \sigma^{2}}\right)_{\mid \sigma^{2}=S_{L}}$. As for the divergence, we have $\left[\nabla_{a} l^{a}\right]_{q}=\left[\left(\partial_{a}+\Gamma_{a b}^{b}\right) l^{a}\right]_{q}$. From

$$
\begin{aligned}
{\left[\Gamma_{a b}^{b}\right]_{q} } & =\frac{1}{2} q^{b c}\left(-\nabla_{c} q_{a b}+2 \nabla_{(a} q_{b) c}\right)+\Gamma_{a b}^{b} \\
& =\frac{1}{2} q^{b c} \nabla_{a} q_{b c}+\Gamma_{a b}^{b}
\end{aligned}
$$

we get

$$
\left[\nabla_{a} l^{a}\right]_{q}=\nabla_{a}\left(\frac{d \lambda}{d \tilde{\lambda}} l^{a}\right)+\frac{1}{2} q^{b c}\left(\nabla_{a} q_{b c}\right) \frac{d \lambda}{d \tilde{\lambda}} l^{a}
$$

This expression openly shows that all differentials are indeed taken on $\Gamma$. Using formula (11) for $q_{a b}$, direct computation gives

$$
\begin{aligned}
{\left[\nabla_{a} l^{a}\right]_{q} } & =\frac{d \lambda}{d \tilde{\lambda}} \nabla_{i} l^{i}+\frac{d}{d \lambda}\left(\frac{d \lambda}{d \tilde{\lambda}}\right)+\frac{1}{2} \frac{d \lambda}{d \tilde{\lambda}}\left\{(D-2) \frac{d}{d \lambda} \ln A_{\gamma}-2 \frac{d}{d \lambda} \ln \alpha_{\gamma}\right\} \\
& =\frac{d \lambda}{d \tilde{\lambda}} \nabla_{i} l^{i}+\frac{1}{2}(D-2) \frac{d \lambda}{d \tilde{\lambda}} \frac{d}{d \lambda} \ln A_{\gamma},
\end{aligned}
$$

where, in the 2nd equality, use of the expression (16) for $\alpha_{\gamma}$ was made. Inserting this into equation (20), we get

$$
[\square f]_{q}=\left\{4+2 \tilde{\lambda} \frac{d \lambda}{d \tilde{\lambda}} \nabla_{i} l^{i}+\tilde{\lambda}(D-2) \frac{d \lambda}{d \tilde{\lambda}} \frac{d}{d \lambda} \ln A_{\gamma}\right\}\left(\frac{d f}{d \sigma^{2}}\right)_{\mid \sigma^{2}=S_{L}} .
$$

Now we are ready to implement condition G3. We require that, if $G=G\left(\sigma_{\mid \tilde{p}^{\prime}}^{2}\right)$, with $\sigma_{\mid \tilde{p}^{\prime}}^{2}=S_{L}$, is solution to $\square G=0$ in $\tilde{p}$ at $\tilde{\lambda}$ on $\gamma\left(\tilde{p}^{\prime}\right.$ is in a (D-dim) neighbourhood of $\tilde{p}$; with $\sigma_{\mid \tilde{p}^{\prime}}^{2} / \sigma_{\mid p^{\prime}}^{2} \rightarrow \tilde{\lambda}^{2} / \lambda^{2}$ when $\tilde{p}^{\prime} \rightarrow \tilde{p}$ and $p^{\prime} \rightarrow p$, due to continuity reasons), i.e. if $\square G_{\mid \tilde{p}}=0$, then $[G]_{q}\left(\sigma^{2}\right) \equiv G\left(S_{L}\left(\sigma^{2}\right)\right)$ be solution of $[\square G]_{q}=0$ in $p$ at $\lambda$ on $\gamma$, i.e.

$$
4+2 \tilde{\lambda} \frac{d \lambda}{d \tilde{\lambda}} \nabla_{i} l^{i}+\tilde{\lambda}(D-2) \frac{d \lambda}{d \tilde{\lambda}} \frac{d}{d \lambda} \ln A_{\gamma}=0
$$

in $p$.

We proceed first to calculate $\square G_{\mid \tilde{p}}$. In $\tilde{p}^{\prime}$, we have

$$
\begin{aligned}
\square G_{\mid \tilde{p}^{\prime}} & =\left(\nabla_{a} \nabla^{a} G\right)_{\mid \tilde{p}^{\prime}} \\
& =\nabla_{a}\left(\left(\partial^{a} \sigma_{\mid \tilde{p}^{\prime}}^{2}\right) \frac{d G}{d \sigma_{\mid \tilde{p}^{\prime}}^{2}}\right) \\
& =\left(\nabla_{a} \partial^{a} \sigma_{\mid \tilde{p}^{\prime}}^{2}\right) \frac{d G}{d \sigma_{\mid \tilde{p}^{\prime}}^{2}}+\left(\partial^{a} \sigma_{\mid \tilde{p}^{\prime}}^{2}\right) \partial_{a} \frac{d G}{d \sigma_{\mid \tilde{p}^{\prime}}^{2}} \\
& =\left(\nabla_{a} \partial^{a} \sigma_{\mid \tilde{p}^{\prime}}^{2}\right) \frac{d G}{d \sigma_{\mid \tilde{p}^{\prime}}^{2}}+\left(\partial^{a} \sigma_{\mid \tilde{p}^{\prime}}^{2}\right)\left(\partial_{a} \sigma_{\mid \tilde{p}^{\prime}}^{2}\right) \frac{d}{d \sigma_{\mid \tilde{p}^{\prime}}^{2}}\left(\frac{d G}{d \sigma_{\mid \tilde{p}^{\prime}}^{2}}\right) .
\end{aligned}
$$


When $\tilde{p}^{\prime} \rightarrow \tilde{p}$ on $\gamma,\left(\partial^{a} \sigma_{\mid \tilde{p}^{\prime}}^{2}\right)\left(\partial_{a} \sigma_{\mid \tilde{p}^{\prime}}^{2}\right) \rightarrow\left(2 \tilde{\lambda} l^{a}{ }_{\mid \tilde{p}}\right)\left(2 \tilde{\lambda} l_{a \mid \tilde{p}}\right)=0$ and thus what matters here is the first term. We have

$$
\begin{aligned}
\nabla_{a} \partial^{a} \sigma_{\mid \tilde{p}^{\prime}}^{2} & =\left(\nabla_{a}\left(2 \lambda l^{a}+2 \nu m^{a}\right)\right)_{\mid \tilde{p}^{\prime}} \\
& =\nabla_{a}\left(2 \tilde{\lambda} l^{a}{ }_{\mid \tilde{p}^{\prime}}+2 \tilde{\nu} m^{a}{ }_{\mid \tilde{p}^{\prime}}\right),
\end{aligned}
$$

where we used of relation (17) and wrote $\tilde{\lambda}=\frac{1}{2}(\tilde{t}+\tilde{r}), \tilde{\nu}=\frac{1}{2}(\tilde{t}-\tilde{r})$. When going to $\gamma$, we get

$$
\begin{aligned}
\left(\nabla_{a} \partial^{a} \sigma^{2}\right)_{\mid \tilde{p}} & =2 \tilde{\lambda}\left(\nabla_{a} l^{a}\right)_{\mid \tilde{p}}+2 l_{\mid \tilde{p}}^{a} \nabla_{a} \tilde{\lambda}+2 m^{a}{ }_{\mid \tilde{p}} \nabla_{a} \tilde{\nu} \\
& =2 \tilde{\lambda}\left(\nabla_{i} l^{i}\right)_{\mid \tilde{p}}+4
\end{aligned}
$$

for $\tilde{\lambda}$ is the affine parameter $\lambda$ at $\tilde{p}$, and $m^{a}{ }_{\mid \tilde{p}}=d x^{a} / d \tilde{\nu}$. Thus, we have

$$
\square G_{\mid \tilde{p}}=\left(2 \tilde{\lambda}\left(\nabla_{i} l^{i}\right)_{\mid \tilde{p}}+4\right) \frac{d G}{d \sigma_{\mid \tilde{p}}^{2}} .
$$

$\square G_{\mid \tilde{p}}=0$ then means

$$
2 \tilde{\lambda}\left(\nabla_{i} l^{i}\right)_{\mid \tilde{p}}+4=0 .
$$

Inserting this into (22), one obtains

$$
-2 \tilde{\lambda}\left(\nabla_{i} l^{i}\right)_{\mid \tilde{p}}+2 \tilde{\lambda} \frac{d \lambda}{d \tilde{\lambda}} \nabla_{i} l^{i}+\tilde{\lambda}(D-2) \frac{d \lambda}{d \tilde{\lambda}} \frac{d}{d \lambda} \ln A_{\gamma}=0
$$

which is

$$
-2 \frac{d \tilde{\lambda}}{d \lambda}\left(\nabla_{i} l^{i}\right)_{\mid \tilde{p}}+2 \nabla_{i} l^{i}+(D-2) \frac{d}{d \lambda} \ln A_{\gamma}=0 .
$$

Thanks to the relation $(\underline{31}, 32]$; see $[34,35])$

$$
\nabla_{a}^{(p)}\left[\Delta(p, P) \nabla_{(p)}^{a} \sigma^{2}(p, P)\right]=2 D \Delta(p, P)
$$

(valid for spacelike/timelike as well as null geodesics), which gives

$$
\nabla_{a} \partial^{a} \sigma^{2}=2 D+\left(\nabla_{a} \ln \Delta^{-1}\right) \partial^{a} \sigma^{2}
$$

with $\partial^{a} \sigma^{2}=2 \lambda l^{a}$ on $\gamma$, using (18) the expansion of the congruence can be usefully expressed in terms of the van Vleck determinant as (cf. [34])

$$
\nabla_{a} l^{a}=\nabla_{i} l^{i}=\frac{D-2}{\lambda}+\frac{d}{d \lambda} \ln \Delta^{-1}
$$

and

$$
\left(\nabla_{a} l^{a}\right)_{\mid \tilde{p}}=\left(\nabla_{i} l^{i}\right)_{\mid \tilde{p}}=\frac{D-2}{\tilde{\lambda}}+\frac{d}{d \tilde{\lambda}} \ln \Delta_{S}^{-1},
$$

where $\Delta_{S}$ is the van Vleck determinant evaluated at $\tilde{p}$.

Substituting this, equation (28) above becomes 


$$
-2\left(\frac{d \tilde{\lambda}}{d \lambda} \frac{D-2}{\tilde{\lambda}}+\frac{d}{d \lambda} \ln \Delta_{S}^{-1}\right)+2\left(\frac{D-2}{\lambda}+\frac{d}{d \lambda} \ln \Delta^{-1}\right)+(D-2) \frac{d}{d \lambda} \ln A_{\gamma}=0
$$

or

$$
-2 \frac{d \tilde{\lambda}}{d \lambda} \frac{1}{\tilde{\lambda}}-\frac{2}{D-2} \frac{d}{d \lambda} \ln \Delta_{S}^{-1}+\frac{2}{\lambda}+\frac{2}{D-2} \frac{d}{d \lambda} \ln \Delta^{-1}+\frac{d}{d \lambda} \ln A_{\gamma}=0
$$

which is

$$
\frac{d}{d \lambda} \ln \left(\frac{\lambda^{2}}{\tilde{\lambda}^{2}}\left(\frac{\Delta_{S}}{\Delta}\right)^{\frac{2}{D-2}} A_{\gamma}\right)=0
$$

Thus

$$
A_{\gamma}=C \frac{\tilde{\lambda}^{2}}{\lambda^{2}}\left(\frac{\Delta}{\Delta_{S}}\right)^{\frac{2}{D-2}}
$$

where $C$ is a constant. To determine $C$, we note that using this expression we get, in the $\lambda \rightarrow \infty$ limit, $A_{\gamma} \rightarrow C$. Since, as we saw, $q_{a b} \rightarrow g_{a b}$ in the same limit implies $A_{\gamma} \rightarrow 1$, we get $C=1$. Our expression for $A_{\gamma}$ is finally

$$
A_{\gamma}=\frac{\tilde{\lambda}^{2}}{\lambda^{2}}\left(\frac{\Delta}{\Delta_{S}}\right)^{\frac{2}{D-2}}
$$

In conclusion, what we have got in this Section is the expression (11) for the qmetric $q_{a b}$ for null geodesics, with the functions $\alpha_{\gamma}$ and $A_{\gamma}$ in it, defined on the null geodesics, required to have the expressions given by equations (16) and (33). We notice that no dependence on the chosen canonical observer is present in $\alpha_{\gamma}$ or $A_{\gamma}$. The expression (11) for $q_{a b}$, however, does depend on the observer, through $m^{a}$.

\section{IV. $\rho$ FOR NULL GEODESICS (LORENTZ SECTOR)}

Using the results of previous Section, let us proceed now to try to find out an expression for $\rho$ for null geodesics. In complete analogy with the timelike/spacelike case, this quantity can be defined, in the Lorentz sector, as (cf. equation (6))

$$
\rho\left(P, l^{a}\right)=\left(\lim _{p \rightarrow P} \frac{\left[d^{D-1} V\right]_{q(g)}(p, P)}{\left[d^{D-1} V\right]_{q(\eta)}(p, P)}\right)_{\gamma\left(l^{a}\right)} .
$$

Here, $\gamma\left(l^{a}\right)$ is a null geodesic through $P$, affinely parameterized through $\lambda=\lambda(p, P)$ with $\lambda(P, P)=0$, with tangent vector $k^{a}=d x^{a} / d \lambda$ along it which takes the value $l^{a}$ at $P$, i.e. $l^{a}=k_{\mid P}^{a}$. The limit is taken for $p$ approaching $P$ along $\gamma\left(l^{a}\right) . d^{D-1} V$ is a $(D-1)$-dim volume element of a null hypersurface $\Sigma_{\gamma}$ through $p$, defined by $\Phi=$ const, with $-\left(\partial_{a} \Phi\right)_{\mid p}=\left(k_{a}\right)_{\mid p}$. Apart from this condition on the gradient, the hypersurface $\Sigma_{\gamma}$ is arbitrary. $\left[d^{D-1} V\right]_{q}$ is the volume of that same element of hypersurface, according to the qmetric, with $\Sigma_{\gamma}$ being null also according to the qmetric $\left(q^{a b} k_{a} k_{b}=0\right.$, as we saw before). The index $q(g)$, or simply $q$, refers to a generic metric $g_{a b}$, while $q(\eta)$ is for the flat case.

$d^{D-1} V$ can be written as follows ([37, 38], e.g.). Using the vector $m^{a}$ as defined in the previous Section, we can write the metric transverse to $k^{a}$ at $p$ as

$$
h_{a b}=g_{a b}+\frac{1}{2}\left(k_{a} m_{b}+m_{a} k_{b}\right) .
$$

Introducing the coordinates $\left(\lambda, \theta^{A}\right)$ for $\Sigma_{\gamma}$, with the coordinates $\theta^{A}$ spanning the $(D-2)$-dim space transverse to the generators of $\Sigma_{\gamma}$, we have the induced metric on the $(D-2)$-dim space is given by 


$$
\begin{aligned}
\sigma_{A B} & =g_{a b} e_{A}^{a} e_{B}^{b} \\
& =h_{a b} e_{A}^{a} e_{B}^{b}
\end{aligned}
$$

in terms of the vectors $e_{A}^{a}=\left(\frac{\partial x^{a}}{\partial \theta^{A}}\right)_{\lambda}\left(e_{A}^{a}\right.$ is orthogonal to both $k^{a}$ and $\left.m^{a}\right)$. The volume element can then be written as

$$
d^{D-1} V=\sqrt{\sigma} d^{D-2} \theta d \lambda
$$

with $\sigma=\operatorname{det}\left(\sigma_{A B}\right)$.

Going to the qmetric, $k^{a}=d x^{a} / d \lambda$ gets mapped to $\left[k^{a}\right]_{q}=d x^{a} / d \tilde{\lambda}=(d \lambda / d \tilde{\lambda}) k^{a}$. $\Sigma_{\gamma}$ is null also according to the qmetric, and the metric transverse (according to $q_{a b}$ ) to $\left[k^{a}\right]_{q}$ is given by

$$
\left[h_{a b}\right]_{q}=q_{a b}+\frac{1}{2}\left(\left[k_{a}\right]_{q}\left[m_{b}\right]_{q}+\left[m_{a}\right]_{q}\left[k_{b}\right]_{q}\right),
$$

with $\left[k_{a}\right]_{q}=q_{a b}\left[k^{a}\right]_{q}=\frac{1}{\alpha_{\gamma}} \frac{d \lambda}{d \lambda} k_{a}=k_{a}$, and $\left[m_{a}\right]_{q}=\frac{d \tilde{\lambda}}{d \lambda} m_{a}$ (to get $q^{a b}\left[k_{a}\right]_{q}\left[m_{b}\right]_{q}=-2$ ). Using the expression (11) for $q_{a b}$, we get

$$
\left[h_{a b}\right]_{q}=A_{\gamma} h_{a b}
$$

and, from $e_{A}^{a}=\left(\frac{\partial x^{a}}{\partial \theta^{A}}\right)_{\lambda}=\left(\frac{\partial x^{a}}{\partial \theta^{A}}\right)_{\tilde{\lambda}}=\left[e_{A}^{a}\right]_{q}$,

$$
\begin{aligned}
{\left[\sigma_{a b}\right]_{q} } & =q_{a b}\left[e_{A}^{a}\right]_{q}\left[e_{B}^{b}\right]_{q} \\
& =\left[h_{a b}\right]_{q}\left[e_{A}^{a}\right]_{q}\left[e_{B}^{b}\right]_{q} \\
& =\left[h_{a b}\right]_{q} e_{A}^{a} e_{B}^{b} \\
& =A_{\gamma} \sigma_{a b} .
\end{aligned}
$$

The qmetric volume element is $\left[d^{D-1} V\right]_{q}=[\sqrt{\sigma}]_{q} d^{D-2} \theta d \tilde{\lambda}=\left[d^{D-2} \mathcal{A}\right]_{q} d \tilde{\lambda}$ with $\left[d^{D-2} \mathcal{A}\right]_{q}=[\sqrt{\sigma}]_{q} d^{D-2} \theta$ the $(D-2)$ $\operatorname{dim}$ area of the element of surface transverse to the generators according to the qmetric, and $d^{D-2} \mathcal{A}=\sqrt{\sigma} d^{D-2} \theta$ the area according to $g_{a b}$. By the way, this form of $\left[d^{D-1} V\right]_{q}$ gives, from

$$
\frac{\left[d^{D-1} V\right]_{q(g)}}{\left[d^{D-1} V\right]_{q(\eta)}}=\frac{\left[d^{D-2} \mathcal{A}\right]_{q(g)}}{\left[d^{D-2} \mathcal{A}\right]_{q(\eta)}},
$$

an equivalent manner, if one wants, to express $\rho$, as

$$
\rho\left(P, l^{a}\right)=\left(\lim _{p \rightarrow P} \frac{\left[d^{D-2} \mathcal{A}\right]_{q(g)}(p, P)}{\left[d^{D-2} \mathcal{A}\right]_{q(\eta)}(p, P)}\right)_{\gamma\left(l^{a}\right)}
$$

From (37),

$$
\begin{aligned}
{\left[d^{D-1} V\right]_{q} } & =[\sqrt{\sigma}]_{q} d^{D-2} \theta d \tilde{\lambda} \\
& =A_{\gamma}^{\frac{D-2}{2}} \sqrt{\sigma} d^{D-2} \theta d \tilde{\lambda} \\
& =A_{\gamma^{\frac{D-2}{2}}} d^{D-2} \mathcal{A} d \tilde{\lambda}
\end{aligned}
$$

Using, on the $(D-2)$-surface, orthogonal coordinates $z^{A}$ such that, chosen any one of them, $z^{\bar{A}}$, it can be put in the form $z^{\bar{A}}=\lambda \chi$, with $\chi$ such that $\lambda d \chi$ is proper distance, we can write

$$
\left[d^{D-1} V\right]_{q}=A_{\gamma}^{\frac{D-2}{2}} \lambda^{D-2}\left(1+\mathcal{O}\left(\lambda^{2}\right)\right)(d \chi)^{D-2} d \tilde{\lambda}
$$


where the $\mathcal{O}\left(\lambda^{2}\right)$ term represents the effects of curvature and is absent in flat case. Substituting here the expression (33) for $A_{\gamma}$, we get

$$
\left[d^{D-1} V\right]_{q}=\tilde{\lambda}^{D-2} \frac{\Delta}{\Delta_{S}}\left(1+\mathcal{O}\left(\lambda^{2}\right)\right)(d \chi)^{D-2} d \tilde{\lambda}
$$

Taking the limit $\lambda \rightarrow 0$ we see that this quantity, as well as $\left[d^{D-2} \mathcal{A}\right]_{q}$, do not vanish, going to the values

$$
\lim _{\lambda \rightarrow 0}\left[d^{D-1} V\right]_{q}=L^{D-2} \frac{1}{\Delta_{L}\left(P, l^{a}\right)}(d \chi)^{D-2} d \tilde{\lambda},
$$

and

$$
\lim _{\lambda \rightarrow 0}\left[d^{D-2} \mathcal{A}\right]_{q}=L^{D-2} \frac{1}{\Delta_{L}\left(P, l^{a}\right)}(d \chi)^{D-2},
$$

with $\Delta_{L}\left(P, l^{a}\right)=\Delta(\bar{p}, P)$, where $\bar{p}$ is that point on the null geodesic $\gamma\left(l^{a}\right)$ which has $\lambda(\bar{p}, P)=L$. In the flat case, $\Delta=1$ identically and then $\Delta_{L}\left(P, l^{a}\right)=1$, as we said, and the expressions above reduces to $\lim _{\lambda \rightarrow 0}\left[d^{D-1} V\right]_{q(\eta)}=$ $L^{D-2}(d \chi)^{D-2} d \tilde{\lambda}$ and $\lim _{\lambda \rightarrow 0}\left[d^{D-2} \mathcal{A}\right]_{q(\eta)}=L^{D-2}(d \chi)^{D-2}$. Thus,

$$
\begin{aligned}
\rho\left(P, l^{a}\right) & =\frac{\lim _{\lambda \rightarrow 0}\left[d^{D-1} V\right]_{q(g)}}{\lim _{\lambda \rightarrow 0}\left[d^{D-1} V\right]_{q(\eta)}} \\
& =\frac{1}{\Delta_{L}\left(P, l^{a}\right)} .
\end{aligned}
$$

We obtain then, in the null case, that same form we found in the timelike/spacelike case. Since $l^{a}$ is assigned with the null geodesic at start, we notice that, even if the qmetric $q_{a b}$ does depend on the chosen observer (through $m^{a}$ ), no dependence on the observer is left in $\rho$.

For timelike/spacelike geodesics, we gave an expansion of $\Delta(p, P)$ in powers of $l=\sqrt{\epsilon \sigma^{2}}$ (equation (8)). For (affinely parameterized) null geodesics, $\Delta(p, P)$ can be analogously expanded in powers of $\lambda$ as ([31]; [33 35])

$$
\Delta(p, P)=1+\frac{1}{6} \lambda^{2} R_{a b}(P) l^{a} l^{b}+o\left(\lambda^{2} R_{a b}(P) l^{a} l^{b}\right)
$$

For $l^{a}$ in a neighbourhood of 0 , this definitely gives

$$
\Delta_{L}\left(P, l^{a}\right)=1+\frac{1}{6} L^{2} R_{a b}(P) l^{a} l^{b}+o\left(L^{2} R_{a b}(P) l^{a} l^{b}\right)
$$

and

$$
\rho\left(P, l^{a}\right)=1-\frac{1}{6} L^{2} R_{a b}(P) l^{a} l^{b}+o\left(L^{2} R_{a b}(P) l^{a} l^{b}\right)
$$

This expression for $\rho$ is analogous to that reported above for timelike/spacelike geodesics (equation (10)), and coincides with the expression which has been found through recourse to Euclidean sector [9] 12 ].

\section{CONCLUSIONS}

Starting from the quantum metric $q_{a b}$ put forward in [1] [3] for timelike/spacelike intervals from the assumption of existence of a lower limit length (along with some consistency conditions), we have introduced a notion of quantum metric $q_{a b}$ for null separated events, and found an expression for it in equation (11) (with (16) and (33)). This expression, and the already existing expressions for timelike and spacelike geodesics [3], complete the task of providing quantum expressions for any kind of spacetime intervals. This quantum metric comes from a single basic request, 
that of length quantization, not from a specific quantum theory of gravity. As such, it finds in principle wide range applicability across any specific quantum model of gravity which foresees quantization of length, i.e. in practice several, if not all, models. This means that in any such model these formulae might be reproducible and cross-checkable.

The formulae for $q_{a b}$ for non-null intervals hint towards a statistical interpretation of spacetime [9], and this is exploited in the introduction of a scalar function $\rho\left(P, v^{a}\right)$ expressing the density of quantum states, at event $P$ in the direction $v^{a}$, associated with atoms we may think spacetime is made of [9-12]. Crucial to this, is the realization that, according to the quantum metric $q_{a b}$ as applied to the Euclidean sector, the cross-sectional area of an equigeodesic surface centered at $P$ does not vanish but goes to a finite limit, when the surface shrinks classically to $P$, signalling this way (quantum) degrees of freedom for spacetime at $P$ [9]. Here, we have used the formula for $q_{a b}$ for null separated events to derive an expression for $\rho$ for $v^{a}$ null, thus remaining entirely within the Lorentz sector, i.e. without making use of Euclideanization (which is how $\rho$ was originally introduced). Key to this, has been to find out that, analogously to what happens in the Euclidean case, according to the null quantum metric the cross-sectional area of a null equi-geodesic surface centered at $P$ does not vanish but remain finite when the surface shrinks classically to $P$. The formula we obtain for $\rho$ turns out to coincide with the formula derived through Euclideanization. The formula for null intervals, joined with the formulae for timelike/spacelike cases, provide a complete account of $\rho$ based on quantum spacetime intervals.

Acknowledgements. I am grateful to Sumanta Chakraborty and Dawood Kothawala for remarks and discussions on the topics of the paper.

[1] D. Kothawala, "Minimal length and small scale structure of spacetime", Phys. Rev. D 88 (2013) 104029, arXiv:1307.5618.

[2] D. Kothawala, T. Padmanabhan, "Grin of the Cheshire cat: Entropy density of spacetime as a relic from quantum gravity", Phys. Rev. D 90 (2014) 124060, arXiv:1405.4967.

[3] D. Jaffino Stargen, D. Kothawala, "Small scale structure of spacetime: van Vleck determinant and equi-geodesic surfaces", Phys. Rev. D 92 (2015) 024046, arXiv:1503.03793

[4] A. Ashtekar, "Introduction to loop quantum gravity and cosmology", Lect. Notes Phys. 863 (2013) 31, arXiv:1201.4598.

[5] T. Thiemann, Modern canonical quantum general relativity (Cambridge University Press, Cambridge, 2007).

[6] C. Rovelli and F. Vidotto, Covariant Loop Quantum Gravity (Cambridge University Press, Cambridge, 2015).

[7] D.N. Coumbe, "Hypothesis on the nature of time", Phys. Rev. D 91 (2015) 124040, arXiv:1502.04320.

[8] D.N. Coumbe, "Quantum gravity without vacuum dispersion", Int. J. Mod. Phys. D 26 (2017) 1750119, arXiv:1512.02519

[9] T. Padmanabhan, "Distribution function of the atoms of spacetime and the nature of gravity", Entropy 17 (2015) 7420 , arXiv: 1508.06286 .

[10] T. Padmanabhan, "Exploring the nature of gravity", arXiv:1602.01474 (2016).

[11] T. Padmanabhan, "The atoms of space, gravity and the cosmological constant", Int. J. Mod. Phys. D 25 (2016) 1630020, arXiv:1603.08658.

[12] T. Padmanabhan, "The atoms of spacetime and the cosmological constant", Journal of Physics: Conf. Series 880 (2017) 012008, arXiv:1702.06136.

[13] T. Padmanabhan, "Dark energy and gravity", Gen. Rel. Grav. 40 (2008) 529, arXiv:0705.2533.

[14] T. Padmanabhan, A. Paranjape, "Entropy of null surfaces and dynamics of spacetime", Phys. Rev. D 75 (2007) 064004, gr-qc/0701003

[15] T. Padmanabhan, "Equipartition of energy in the horizon degrees of freedom and the emergence of gravity", Mod. Phys. Lett. A 25 (2010) 1129, arXiv:0912.3165.

[16] T. Padmanabhan, "Surface density of spacetime degrees of freedom from equipartition law in theories of gravity", Phys. Rev. D 81 (2010) 124040, arXiv:1003.5665.

[17] D. Kubiznak, R.B. Mann, M. Teo, "Black hole chemistry: thermodynamics with Lambda", Class. Quantum Grav. 34 (2017) 063001, arXiv:1608.06147.

[18] D. Kubiznak, R.B. Mann, "P-V criticality of charged AdS black holes", JHEP 1207 (2012) 033, arXiv:1205.0559.

[19] A.F. Vargas, E. Contreras, P. Bargueño, "SAdS black holes and spacetime atoms: a heuristic approach", Gen. Rel. Grav. 50 (2018) 117, arXiv:1712.01159.

[20] M. Visser, "How to Wick rotate generic curved spacetime", arXiv:1702.05572 (1991).

[21] D. Kothawala, "Action and observer dependence in Euclidean quantum gravity", Class. Quantum Grav. 35 (2018) 03LT01, arXiv:1705.02504.

[22] J. Greensite "Dynamical origin of the Lorentzian signature of spacetime", Phys. Lett. B 300 (1993) 34, gr-qc/9210008

[23] A. Carlini, J. Greensite, "Why is spacetime Lorentzian?" Phys. Rev. D 49 (1994) 866, gr-qc/9308012

[24] A. White, S. Weinfurtner, M. Visser, "Signature change events: A challenge for quantum gravity ?", Class. Quantum Grav. 27 (2010) 045007, arXiv:0812.3744.

[25] R.D. Sorkin, "Is the spacetime metric Euclidean rather than Lorentzian ?", in Recent Research in Quantum Gravity, edited by A. Dasgupta (Nova Science Publishers, New York, 2013), arXiv:0911.1479.

[26] J. Ambjørn, J. Jurkiewicz, R. Loll, "Quantum gravity, or the art of building spacetime", in Approaches to quantum gravity, edited by D. Oriti, (Cambridge University Press, Cambridge, 2009), hep-th/0604212 
[27] A. Pesci, "Spacetime atoms and extrinsic curvature of equi-geodesic surfaces", arXiv:1511.08665 (2015).

[28] J.L. Synge, Relativity: The general theory (North-Holland, Amsterdam, 1960).

[29] J.H. van Vleck, "The correspondence principle in the statistical interpretation of quantum mechanics", Proc. Nat. Acad. Sci. USA 14 (1928) 178.

[30] C. Morette, "On the definition and approximation of Feynman's path integrals", Phys. Rev. 81 (1951) 848.

[31] B.S. DeWitt, R.W. Brehme, "Radiation damping in a gravitational field", Annals Phys. 9 (1960) 220.

[32] B.S. DeWitt, The dynamical theory of groups and fields (Gordon and Breach, New York, 1965).

[33] S.M. Christensen, "Vacuum expectation value of the stress tensor in an arbitrary curved background: The covariant point-separation method", Phys. Rev. D 14 (1976) 2490.

[34] M. Visser, "van Vleck determinants: geodesic focussing and defocussing in Lorentzian spacetimes", Phys. Rev. D 47 (1993) 2395, hep-th/9303020

[35] E. Poisson, A. Pound, I. Vega, "The motion of point particles in curved spacetime", Liv. Rev. Rel. 14 (2011) 7 , arXiv:1102.0529

[36] D. Kothawala, "Intrinsic and extrinsic curvatures in Finsleresque spaces", Gen. Rel. Grav. 46 (2014) 1836, arXiv:1406.2672.

[37] E. Poisson, A relativist's toolkit (Cambridge University Press, Cambridge, 2004).

[38] T. Padmanabhan, Gravitation: Foundations and frontiers (Cambridge University Press, Cambridge, 2010). 